\title{
Homology-dependent recombination of large synthetic pathways into E. coli genome via $\lambda$-Red and CRISPR/Cas9 dependent selection methodology
}

\author{
Buli Su, Dandan Song and Honghui Zhu* (i)
}

\begin{abstract}
Background: Metabolic engineering frequently needs genomic integration of many heterologous genes for biosynthetic pathway assembly. Despite great progresses in genome editing for the model microorganism Escherichia coli, the integration of large pathway into genome for stabilized chemical production is still challenging compared with small DNA integration.

Results: We have developed a $\lambda$-Red assisted homology-dependent recombination for large synthetic pathway integration in E. coli. With this approach, we can integrate as large as $12 \mathrm{~kb}$ DNA module into the chromosome of $E$. coli W3110 in a single step. The efficiency of this method can reach 100\%, thus markedly improve the integration efficiency and overcome the limitation of the integration size adopted the common method. Furthermore, the limiting step in the methylerythritol 4-phosphate (MEP) pathway and lycopene synthetic pathway were integrated into the W3110 genome using our system. Subsequently, the yields of the final strain were increased 106 and 4.4-fold compared to the initial strain and the reference strain, respectively.
\end{abstract}

Conclusions: In addition to pre-existing method, our system presents an optional strategy for avoiding using plasmids and a valuable tool for large synthetic pathway assembly in E. coli.

Keywords: Metabolic engineering, Chromosomal integration, Lambda Red, CRISPR-Cas9, Escherichia coli

\section{Background}

Escherichia coli is a model microorganism usually used for synthetic biology and industrial applications [1]. Meanwhile, E. coli is known as one of the most promising host for the development of microbial cell factories [2]. Over the last decade, various metabolic engineering strategies, including overexpression of key genes,

\footnotetext{
*Correspondence: zhuhh@gdim.cn

State Key Laboratory of Applied Microbiology Southern China, Guangdong Provincial Key Laboratory of Microbial Culture Collection and Application, Guangdong Microbial Culture Collection Center (GDMCC), Guangdong Institute of Microbiology, Guangdong Academy of Sciences, Guangzhou 510070, People's Republic of China
}

deletion of competitive pathways and chromosomal integrations, have been developed in E. coli to improve the metabolic flux and consequently increase production yields [3]. Novel approaches for introducing synthetic DNA modules, particularly large synthetic pathways, into $E$. coli would therefore greatly facilitate engineering processes.

The widely used methods for genome editing in $E$. coli were developed on the basis of $\lambda$-Red promoted homology-dependent recombination (HDR). Chromosomal integration of DNA modules with the size of about 2000 bp could be accomplished through $\lambda$-Red promoted HDR with high efficiency [4]. However, the recombination efficiency could decrease drastically for large DNA

(c) The Author(s) 2020. This article is licensed under a Creative Commons Attribution 4.0 International License, which permits use, sharing, adaptation, distribution and reproduction in any medium or format, as long as you give appropriate credit to the original author(s) and the source, provide a link to the Creative Commons licence, and indicate if changes were made. The images or other third party material in this article are included in the article's Creative Commons licence, unless indicated otherwise in a credit line to the material. If material is not included in the article's Creative Commons licence and your intended use is not permitted by statutory regulation or exceeds the permitted use, you will need to obtain permission directly from the copyright holder. To view a copy of this licence, visit http://creativecommons.org/licenses/by/4.0/. The Creative Commons Public Domain Dedication waiver (http://creativecommons.org/publicdomain/zero/1.0/) applies to the data made available in this article, unless otherwise stated in a credit line to the data. 
modules ( $>2000 \mathrm{bp}$ ) and chromosomal integration of DNA module which was larger than $2500 \mathrm{bp}$ using $\lambda$-Red promoted HDR was very difficult [5]. In particular, the elimination of antibiotic marker was inconvenient, and the residual FRT sites might bring about unexpected recombination in the genome of the edited strain. Various chromosomal integration strategies based on $\lambda$-Red system have been established for large DNA modules integration, including I-SceI cleavage-facilitated recombination [6], knock-in/knock-out (KIKO) vector mediated integration [7] and pSB1K3(FRTK) vector aided insertion [8]. However, the integration efficiency was significantly decreased when the size of the DNA modules was gradually increased. Currently, the maximum length of the integrated DNA modules was about $10 \mathrm{~kb}$ assisted by the $\lambda$-Red promoted HDR [5]. As an exceptional case, DNA modules $(\sim 15 \mathrm{~kb})$ were divided into four segments (each $\sim 3 \mathrm{~kb}$ ) and then iteratively integrated into $E$. coli genome [9]. Furthermore, a $50 \mathrm{~kb}$ DNA module from $B$. subtilis 168, divided into seven segments (each $\sim 6-7 \mathrm{~kb}$ ), was inserted into $E$. coli genome through iterative integration [9]. This method could integrate large DNA fragment, which however required many rounds of integration to achieve large synthetic pathways integration.

In recent years, CRISPR/Cas9 has become the most widely used technology for genome editing in a variety of organisms [10]. In E. coli, the CRISPR-Cas9 technology was generally accomplished assisted by the $\lambda$-Red promoted HDR for chromosomal integration [11]. For example, DNA modules as large as $7 \mathrm{~kb}$ could be integrated in E. coli chromosome with $>60 \%$ efficiency using CRISPRbased technique [12]. Another example, the $10 \mathrm{~kb}$ isobutanol biosynthetic pathways were able to scarlessly insert into the genome with an efficiency of 50\% [13]. However, the efficiencies and the fragments size of these integrations do not meet the requirement of some engineering goals.

In this study, we developed a new strategy for integration of large synthetic pathways into E. coli W3110 genome (Fig. 1). To achieve this, we harnessed $\lambda$-Red and CRISPR/Cas9 system to increase recA-mediated HDR efficiency and delete redundant sequences, respectively. To demonstrate the feasible with which this system could be applied to genetical applications, we integrated the optimized synthetic pathways which combined limiting step in MEP pathway and lycopene synthetic pathway into E. coli W3110 genome using our system (Fig. 2). This approach enabled integration of synthetic pathways as large as $12 \mathrm{~kb}$ with efficiency of $100 \%$. Consequently, we were able to obtain a strain capable of producing lycopene in a single step and the yields were increased 106 and 4.4-fold compared to the initial strain and the reference strain, respectively. Concerning the integrated DNA fragment size and the integrated efficiency, we ensured that our platform will be useful for metabolic engineering and synthetic biology in E. coli.

\section{Results \\ Design of the $\lambda$-Red-assisted homology-dependent recombination for large synthetic pathway integration}

The workflow of present strategy was illustrated in Fig. 1. The genome editing procedure was performed via plasmid pRC-IS5 and pCas. The plasmid pRC-IS5 contained the fragmentary IS5 sequence, the narrow-host-range replicon $\mathrm{R} 6 \mathrm{~K}$, the chloramphenicol marker, the large synthetic pathways and a gRNA recognition region (N20PAM). The N20PAM sequence from Saccharomyces cerevisiae was used for reducing the off-target frequency. The plasmid pCas containing the $\lambda$-Red and CRISPR/ Cas9 system [14]. When conducted the editing processes, the vector pRC-IS5 was inserted into the E. coli W3110 genome by recA-mediated HDR assisted by $\lambda$-Red. Then a Double-stranded break (DBS) which induced $\lambda$-Red promoted HDR by donor template $(90$ bp synthetically single-stranded primer) was created by Cas 9 nuclease to accomplish the editing processes (Fig. 1). Finally, the redundant sequences including IS5 sequence, chloramphenicol marker and R6K were deleted.

To facilitate this platform for genome editing, $\lambda$-Red recombinases (Exo, Beta, and Gam) were expressed to facilitate the recA-mediated HDR. The plasmid pCas (MolecularCloud Cat. No.: MC_0000011) was used to fulfill this function, in which $\lambda$-Red was induced via the inducible promoters $p B A D$ and the CRISPR/Cas9 systems was controlled by the native promoter. The $\lambda$-Red system was induced by $0.2 \% \mathrm{~L}$-arabinose for the chromosomal insertion of the pRC-IS5, while the CRISPR/Cas9 system was expressed for the generation of a DSB at the universal N20PAM to remove the redundant sequences.

\section{Construction of a model synthetic pathway for integration}

The biosynthesis of lycopene was extensively studied and the synthetic pathway for lycopene was usually used as the model pathway in metabolic engineering and synthetic biology $[15,16]$. Thus, the production of lycopene was chosen as a model pathway in this work. Many studies have demonstrated that the first two and last two steps of the MEP pathway were the limiting steps for lycopene production [17-20]. Based on the metabolic regulation of MEP pathway [21] (Fig. 2a), we divided the model synthetic pathways into three modules which comprised feedforward module including $d x s$ (Gene ID: 938609) and $d x r$ (Gene ID: 939636), feedback module including idi (Gene ID: 938985) and crtE and lycopene synthetic module including crtI, crtE and crtB (GenBank: CP002191) (Fig. 2b). Many previous 


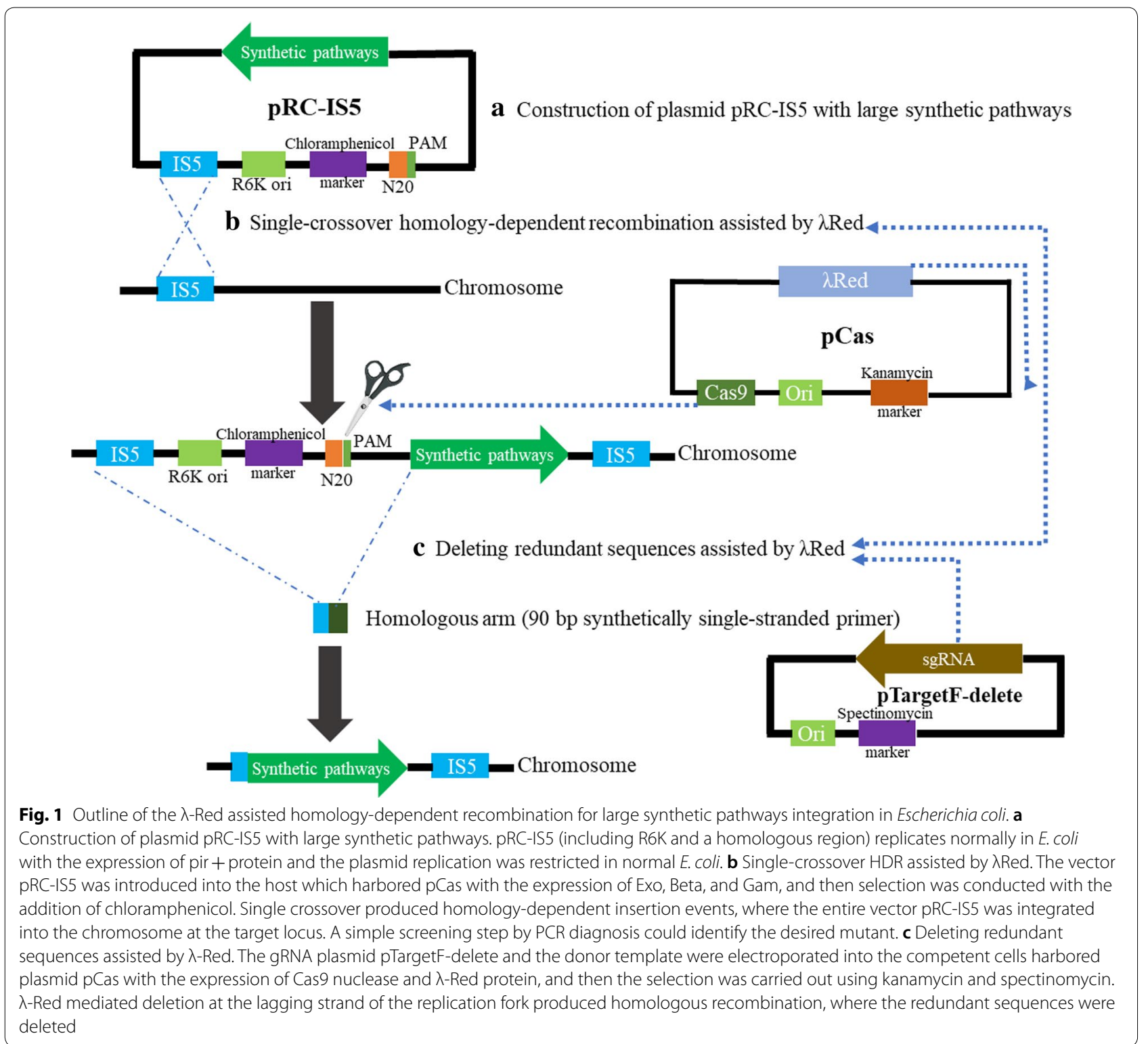

studies have proved that the carbon and energy flux were directed to cell growth in the early growth phase and later redirected to synthetic pathways to support target product formation in growth regulated pathways $[22,23]$. The transcriptome analysis along with the growth phase has been done by a previous work [24]. In order to construct growth regulated pathways, we have picked the promoters which maintained low expression at exponential phase and strong upregulated when cultured to the end of exponential phase and held high expression latterly for these three modules based on the transcriptome data along with the growth phase (GSE102672). We defined each promoter as the $600 \mathrm{bp}$ upstream of the ribosome-binding site (RBS) of its corresponding coding sequence, since these regions generally contained most regulatory sequences [25]. To avoid the influence by RBS site, the same Shine-Dalgarno sequence was used for each module.

As shown in Fig. 3, the $\operatorname{trc}$ (from pTrc99a [26]) was the best promoter for lycopene synthetic module, indicating that stronger promoter was needed for lycopene production. Similar result from previous work suggested that efficient lycopene production relied on maintaining high levels of lycopene synthase [27]. Based on the shake flask fermentations, the PphoR and PyejG were the best promoters for feedforward and feedback modules, 

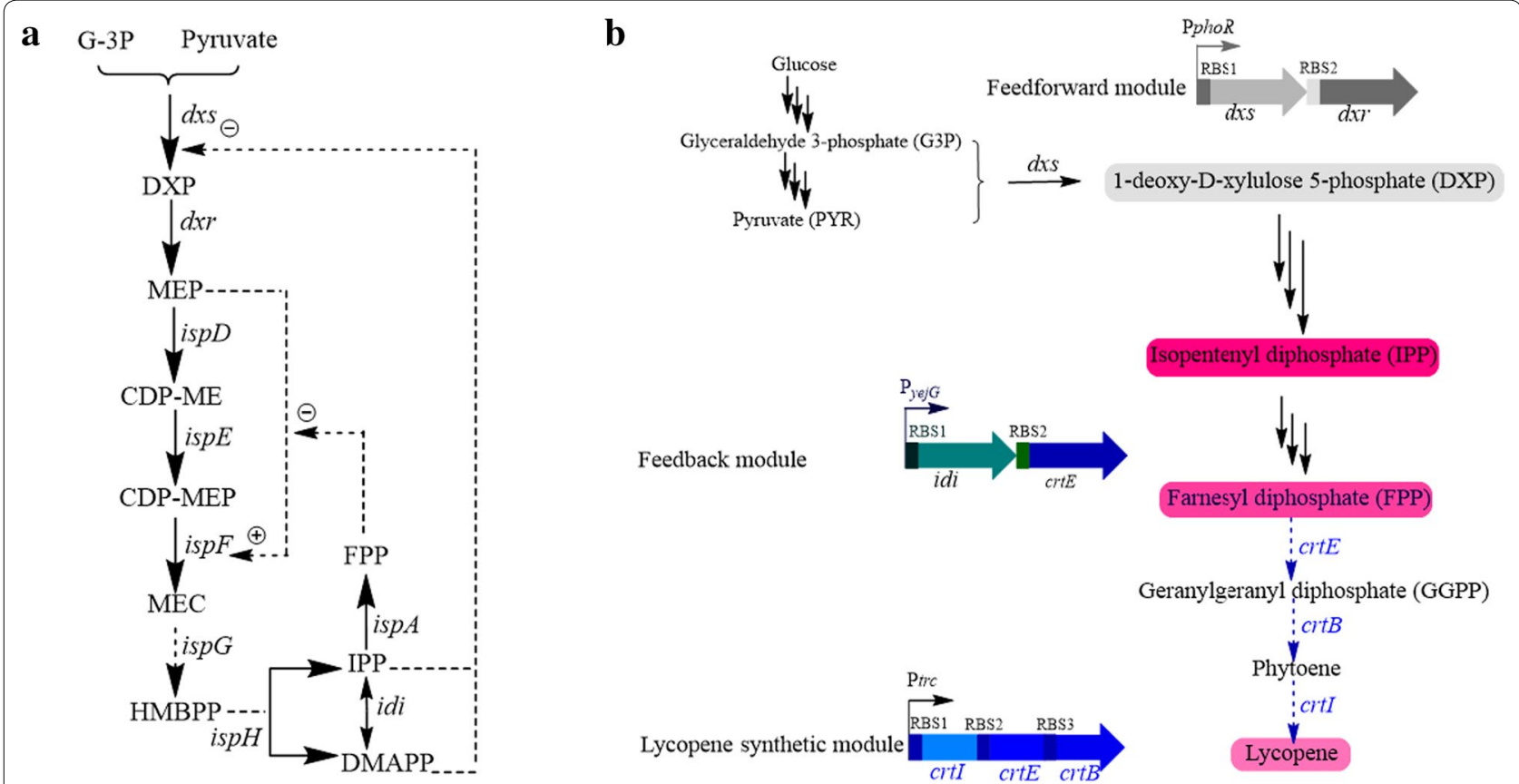

Fig. 2 a MEP pathway and related metabolism showing the major metabolic regulatory points. D-glyceraldehyde 3-phosphate (G-3P);1-deoxy-D-xylulose 5-phosphate (DXP); methylerythritol 4-phosphate (MEP); diphosphocytidyl methylerythritol (CDP-ME); diphosphocytidyl methylerythritol 2-phosphate (CDP-MEP); hydroxymethylbutenyl diphosphate (HMBPP); isopentenyl diphosphate (IPP); dimethylallyl diphosphate (DMAPP); farnesyl diphosphate (FPP). b The limiting step for lycopene production was divided into three modules. The feedforward module including $d x s$ and $d x r$, feedback module including idi and crtE and lycopene synthetic module including $c r t$, crtE and crtB for lycopene production

respectively. Thus, promoters trc, PphoR and PyejG were chosen for the construction of the model synthetic pathways.

\section{Integration of a $12 \mathrm{~kb}$ DNA module into E. coli W3110 genome}

To verify the efficacy of the designed platform for integration of large fragments, we used the above synthetic pathways $(\sim 12 \mathrm{~kb})$ as a model module to integrate it into E. coli W3110 genome. We divided the plasmid pRC-IS5 into four segments including three modules and the vector backbone. Firstly, we obtained the integrative vector pRC-IS5 through Gibson assembly method (Additional file 1: Fig. S1) [28]. Subsequently, pRC-IS5 was integrated into the IS5 locus through recA-mediated HDR assisted by $\lambda$-Red. Consequently, the optimized lycopene synthetic pathways $(\sim 12 \mathrm{~kb})$ was integrated into $E$. coli W3110 genome. The correct integration was verified by the red color and colony PCR, and the edited strain was designated as EC-IS5. We found that all the colonies on the plates were red colored with $\lambda$-Red and there was no colony without adding arabinose to induce $\lambda$-Red (Additional file 1: Fig. S2). Then the red colonies were further verified by colony PCR (Fig. 4c). This result indicated that the $\lambda$-Red system was crucial for recA-mediated HDR when generated large pathway integration. Strain EC-IS5 produced $9 \mathrm{mg} / \mathrm{g}$ CDW of lycopene in the shake flask fermentation, whereas the plasmid-based strain EC101 and EC401 produced 0.086 and $2.1 \mathrm{mg} / \mathrm{g}$ CDW of lycopene, respectively (Fig. 5). EC-IS5 produced 105-fold increase of lycopene yield through integrating the optimized lycopene synthetic pathways into genome compared with the initial strain (EC101). These results indicated that this strategy which combined modular pathway engineering and integrated strategy represented a remarkable synergy.

Deletion of redundant sequences with CRISPR-Cas9 system We next cultivated EC-IS5 in medium supplemented with kanamycin at $30{ }^{\circ} \mathrm{C}$ to maintain the pCas plasmid and made the competent cell washed by $10 \%$ glycerol. To obtain marker-free strains, the competent EC-IS5 was transformed with 90 bp synthetically singlestranded donor template and plasmid pTargetF-delete (constructed based on pTargetF-cadA [14]) using electroporation and then were spread on the LB plate with kanamycin and spectinomycin. The deletion efficiency of the redundant sequences was exceeded $70 \%$ after an overnight incubation (Additional file 1: Fig. S3). This feature might facilitate iterative genome editing. Then the 

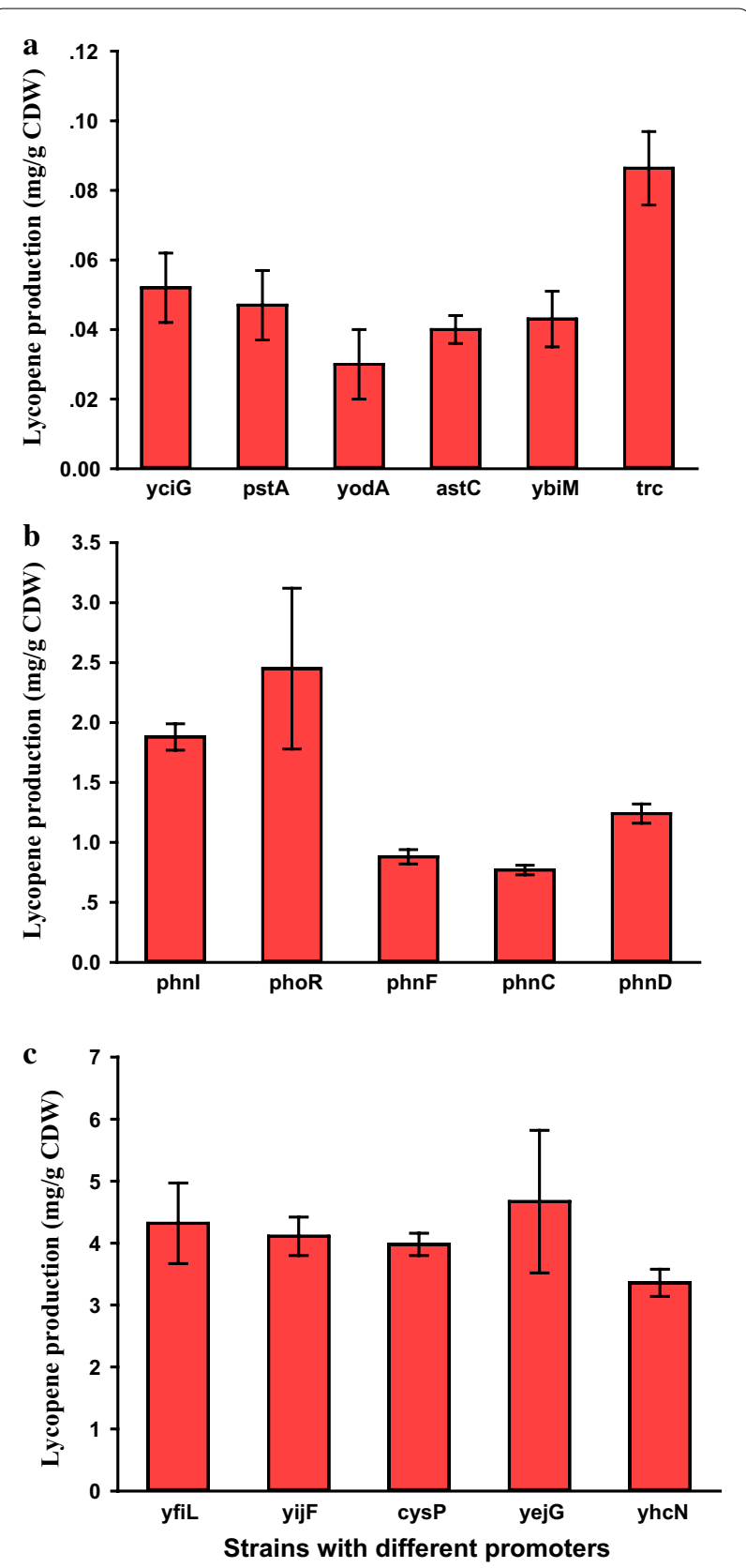

Fig. 3 Promoters characterization of three modules for lycopene biosynthesis. a Selection promoters for lycopene synthetic modules (including crtl, crtE and crtB). Lycopene synthetic modules were overexpressed in E. coli W3110 with the native MEP pathway. trc, EC101; yciG, EC102; pstA, EC103; yodA, EC104; astC, EC105; ybiM EC106. $\mathbf{b}$ Selection promoters for feedforward modules (including $d x s$ and $d x r$. Feedforward modules were overexpressed along with lycopene synthetic modules. phnl, EC201; phoR, EC202; phnF, EC203; phnC, EC204; phnD, EC205. c Selection promoters for feedback modules (including idi and $c r t E$ ). Feedback modules were overexpressed along with lycopene synthetic modules. yfiL, EC301; yijF, EC302; cysP, EC303; yeiG, EC304; yhcN, EC305. Each value represents the average \pm SD of three biological replicates final strain EC-IS5 $(\Delta \mathrm{Cm})$ was used for lycopene production without antibiotic maintenance. As shown in Fig. 5, strain EC-IS5 $(\Delta \mathrm{Cm})$ produced $9.1 \mathrm{mg} / \mathrm{g} C D W$ lycopene, which was 4.4-fold of the reference strain (EC401). This result confirmed that chromosomal integration shown great advantage than plasmid-based method.

\section{Discussion}

In a previous study, we developed a platform for chromosomal integration $(\sim 1.5 \mathrm{~kb})$ in E. coli for xylitol production using recA-mediated HDR [29]. In preliminary experiment before this study, we attempted to integrate a larger pathway ( $7 \mathrm{~kb}$, only including $\operatorname{crtI}, \operatorname{crtB}$ and $\operatorname{crt} E)$ into $E$. coli chromosome using recA-mediated HDR. Nevertheless, we could not get any colonies using the above method and CRISPR/Cas9 system [14]. AlonsoGutierrez and colleagues attempted to integrate a synthetic pathway comprised terpene synthase $(\sim 12 \mathrm{~kb})$ into E. coli DH1 genome using assistant plasmid which could mediate chromosomal integration by $\lambda$-Red promoted HDR [7]. However, they could not get the expected integration through this system. They claimed that the large size of the synthetic pathway and the complicacy of the pathway might be the most probable explanation for these failing attempts. As an alternative, they divided the synthetic pathway into three segments (shorter than $5 \mathrm{~kb}$ ) and integrated them through three rounds of integration to achieve the final integration [30].

Escherichia coli was highly dependent on a homologous recombination to repair DBS in the chromosome. $\lambda$-Red promoted HDR successfully supplemented the low efficiency of the $E$. coli native repair system and, thus, succeeded in genome editing, while single DSB could not be repaired without $\lambda$-Red [14]. The recA-mediated HDR is another form of allelic exchange [31]. However, this recombination is insufficient for large pathway integration (Fig. 4b). Fortunately, we successfully rescued the low efficiency of recA-mediated HDR by using $\lambda$-Red. RecA is one of DNA strand exchange proteins which are essential for homologous recombination. In vivo, RecA preferentially binds to ssDNA (double stranded DNA (dsDNA) breaks or ssDNA gaps in replication forks stall), and then the assembly of a presynaptic filament of RecA on the ssDNA was generated during homologous recombination, which in turn uses the ssDNA sequence to search for a homologous region in the dsDNA [32]. Meanwhile, the $\lambda$-Red system consists of several genetic components (Exo, Beta, and Gam) and Beta also binds to the ssDNA [2]. Although we do not know what is the real mechanism, we speculated that Beta would likely facilitate rescuing the low activity of recA-mediated HDR for large synthetic pathway. 


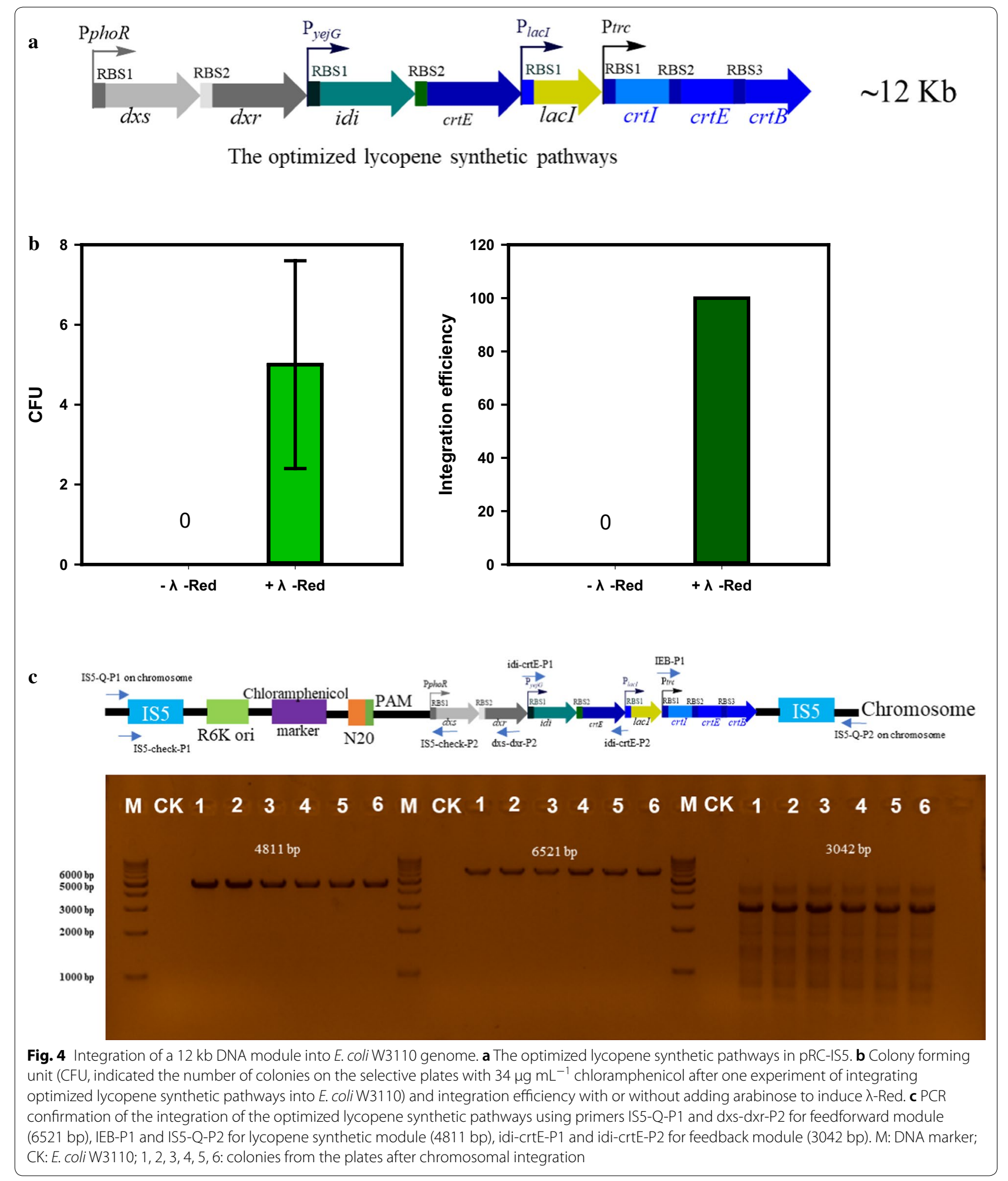




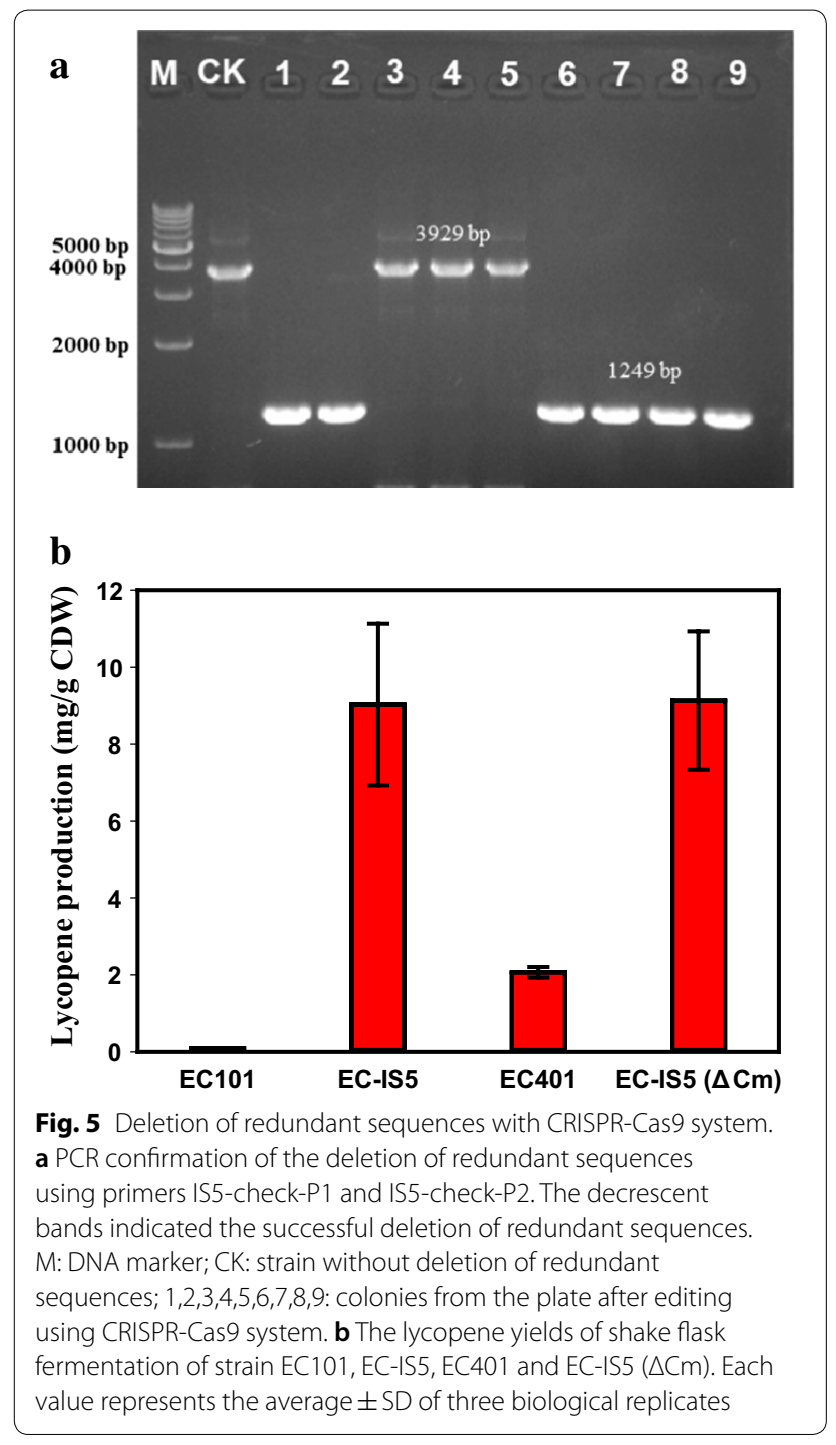

Recent years, CRISPR/Cas9 based genome editing has been obtained great progresses in E. coli. However, chromosomal integration of large DNA modules was still limited by low efficiency and integration size compared with chromosomal integration of DNA modules shorter than $2000 \mathrm{bp}$. To alleviate these issues, Li and colleagues developed a platform with multiple step integration of divided segments [33]. They found that the integration efficiency decreased gradually with the increasing size of the modules, and the efficiency for a $7 \mathrm{~kb}$ DNA module was about $10 \%$ which was similar to that in E. coli MG1655 [34]. To facilitate the high efficiency and the preparation of the DNA modules, about 3-4 kb of the entire DNA module was optimal. They could insert a $15.4 \mathrm{~kb}$ synthetic pathway which contained several crucial genes for uridine biosynthesis into E. coli W3110 genome with five steps of integration [33]. However, this strategy needed many rounds of integration to access the final goals.

Using our platform, we were able to obtain a strain capable of producing lycopene in a single step and the production yields were increased 106 and 4.4-fold compared to the initial strain (EC101) and the reference strain (EC401), respectively (Additional file 1: Fig. S4). This study clearly demonstrate that our platform was quite feasible and useful for constructing microbial cell factories which needed large synthetic pathways. Therefore, we concluded that the recA-mediated HDR integration aided by $\lambda$-Red in this study was relatively practical for metabolic engineering (Table 1). Furthermore, we speculated that our platform facilitated integration of large synthetic pathways could be applied to other prokaryotic microorganism to achieve stable strains for chemical production, for that insertion sequences were widely distributed in many microorganism [35].

\section{Conclusions}

We have developed a useful platform for integration of large synthetic pathways into E. coli W3110 genome. Taking advantage of the $\lambda$-Red promoted HDR and the Cas 9 nuclease, only the integrative vector is needed to construct for each round of integration. Another characteristic is that stable strains can be obtained by integration of large synthetic pathways that are responsible for valuable chemicals biosynthesis. In order to verify the feasibility of our platform, a $12 \mathrm{~kb}$ DNA module contained several key genes for lycopene biosynthesis was integrated into the E. coli W3110 chromosome. The production yields were increased 106 and 4.4-fold compared to the initial strain (EC101) and the reference strain (EC401), respectively. Our platform has been proven to be practical in $E$. coli and would be adapted for the production of valuable chemicals.

\section{Methods}

Strains and culture medium

Strains and plasmids used in this study are listed in Table 2. E. coli strain DH5 $\alpha$ and DH5 $\alpha$ pir $\left(\right.$ pir $^{+}$for propagating $\mathrm{R} 6 \mathrm{~K}$ ori) were used for the construction of the plasmids. E. coli W3110 was used for chromosomal integration. Strains were cultured in Luria-Bertani (LB) medium supplemented with $100 \mu \mathrm{g} \mathrm{mL} \mathrm{m}^{-1}$ ampicillin,

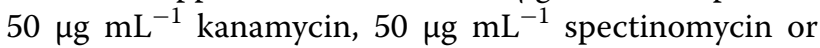
$34 \mu \mathrm{g} \mathrm{mL}{ }^{-1}$ chloramphenicol when needed at $37^{\circ} \mathrm{C}$ or $30{ }^{\circ} \mathrm{C}$ with shaking at $200 \mathrm{rpm}$.

\section{Plasmid construction}

Primers for construction of various plasmids are listed in Additional file 1: Table S1. Plasmids for expression of 
Table 1 Methods for the integration of large DNA module into $E$. coli chromosome

\begin{tabular}{|c|c|c|c|c|c|c|c|}
\hline Method & Technique feature & $\begin{array}{l}\text { Integration } \\
\text { efficiency } \\
(\%)\end{array}$ & Integration sites & $\begin{array}{l}\text { Maximum } \\
\text { integration } \\
\text { size (kb) }\end{array}$ & $\begin{array}{l}\text { Rounds } \\
\text { of integration }\end{array}$ & $\begin{array}{l}\text { Marker left or } \\
\text { markerless }\end{array}$ & Reference \\
\hline I-Scel endonuclease & $\begin{array}{l}\text { Combined } \lambda \text {-Red and } \\
\text { I-Scel Cleavage }\end{array}$ & $19.2-100$ & Desired locus & 7 & One & Markerless & [5] \\
\hline$\lambda$-Red recombination & $\lambda$-Red assisted & $0-50$ & Desired locus & 7.3 & One & Markerless & {$[7]$} \\
\hline \multirow[t]{2}{*}{$\lambda$-Red recombination } & \multirow[t]{2}{*}{$\begin{array}{l}\text { Combined } \lambda \text {-Red and } \\
\text { CRISPR/Cas9 }\end{array}$} & 10 & Desired locus & 7 & One & Markerless & $\begin{array}{l}{[33]} \\
{[9]}\end{array}$ \\
\hline & & 60 & & 15.4 & Five & Markerless & \\
\hline \multirow[t]{2}{*}{$\lambda$-Red recombination } & \multirow[t]{2}{*}{$\lambda$-Red assisted } & Not provide & flik & 15 & Four & Markerless & \\
\hline & & Not provide & flik & 50 & Seven & Markerless & \\
\hline CRISPR/Cas9 & $\begin{array}{l}\text { Combined } \lambda \text {-Red and } \\
\text { CRISPR/Cas9 }\end{array}$ & 60 & Desired locus & 7 & One & Markerless & [12] \\
\hline CRISPR/Cas9 & $\begin{array}{l}\text { Combined } \lambda \text {-Red and } \\
\text { CRISPR/Cas9 }\end{array}$ & 50 & Desired locus & 10 & One & Markerless & [13] \\
\hline $\begin{array}{l}\text { RecA homologous } \\
\text { recombination }\end{array}$ & $\begin{array}{l}\text { Combined } \lambda \text {-Red, RecA } \\
\text { and CRISPR/Cas9 }\end{array}$ & 100 & 155 & 12 & One & Markerless & This study \\
\hline
\end{tabular}

heterologous lycopene synthesis pathway are based on pCDFDuet-1 or pET-30a-trc, plasmids for expression of feedforward module and feedback module were based on pACYCDuet-1. Plasmid containing the large synthetic pathways is divided into several small fragments, including three modules and the vector backbone. All the plasmids were constructed according to the protocol of ClonExpress MultiS One Step Cloning Kit (Vazyme, China).

\section{Chromosomal integration procedure}

Briefly, the host strain E. coli W3110 was transformed with pCas and then was prepared for competent cells with $\lambda$-Red recombinase induction by L-arabinose according to the protocol [36]. Immediately, $100 \mu \mathrm{L}$ of the competent cells was mixed with $300 \mathrm{ng}$ of plasmid pRC-IS5 in MicroPulser (Eppendorf). After electroporation $(2.5 \mathrm{kV}, 5 \mathrm{~ms})$, the competent cells were suspended in $1 \mathrm{~mL} \mathrm{LB}$ broth quickly. After $6 \mathrm{~h}$ incubated at $30{ }^{\circ} \mathrm{C}$, cells were centrifuged and resuspended in $0.1 \mathrm{~mL}$ sterile water. Then the cells were spread on LB agar plates with chloramphenicol. After genome editing, the colonies on the plates were identified by red color and colony PCR with primers IS5-Q-P1, dxs-dxr-P2, idi-crtE-P1, idi-crtE-P2, IEB-P1 and IS5-Q-P2 which straddling the synthetic pathway on chromosome (Additional file 1: Table S1). The editing efficiency was calculated as the number of colonies with red color divided by the number of all the colonies. The correct strain was transferred into LB broth with kanamycin, and was prepared as electrocompetent cells with the expression of Cas9 nuclease and $\lambda$-Red proteins. Donor template (90 bp synthetically single-stranded primer) and plasmid pTarget-delete (including the gRNA sequence) were electroporated into the competent cells and then the cells were spread on the LB plate with kanamycin and spectinomycin. Deletion of the redundant sequences was identified by chloramphenicol sensitive and colony PCR using primers IS5-check-P1 and IS5-check-P2 (Additional file 1: Table S1).

\section{Shake flask cultures and analysis of lycopene}

For shake flask fermentation, a single colony selected from a fresh LB agar plate was grown overnight in $5 \mathrm{~mL}$ of LB broth in a shaker at $37{ }^{\circ} \mathrm{C}$ for overnight growth, then $1 \mathrm{~mL}$ of the preculture was inoculated into $250 \mathrm{~mL}$ shake flask containing $50 \mathrm{~mL} 2 \times \mathrm{TY}$ medium with $4 \%$ glycerol and grown at $30{ }^{\circ} \mathrm{C}$ for $48 \mathrm{~h}$. Individual flasks were stopped at regular times to determine biomass and lycopene yields. Extraction of carotenoid was as described by literature with some modifications [37]. Briefly, cells were harvested by centrifugation at $8000 \mathrm{~g}$ for $5 \mathrm{~min}$, and then were suspended in $1 \mathrm{~mL}$ of acetone. The lysate was incubated at $55^{\circ} \mathrm{C}$ for $15 \mathrm{~min}$ and centrifuged at $12,000 \mathrm{~g}$ for $20 \mathrm{~min}$. The acetone supernatant was transferred into a clean tube for measuring lycopene. The lycopene content of the extracts was determined by UV/Vis spectrometer (PerkinElmer Lambda 45) at $470 \mathrm{~nm}$. Spectra was recorded in acetone using an A $1 \% 1 \mathrm{~cm}$ of 3450 [38]. The yields of lycopene were expressed as mg per $\mathrm{g}$ cell dry weight (mg/g CDW). 
Table 2 Escherichia coli strains and plasmids used in this study

\begin{tabular}{|c|c|c|}
\hline Strain/plasmid & Description & Source \\
\hline \multicolumn{3}{|l|}{ Strains } \\
\hline $\mathrm{DH} 5 \mathrm{a}$ & supE44 $\triangle$ lacU169 ( $(80$ lacZDM15) hsdR17 recA1 endA1 gyrA96 thi-1 relA1 & Invitrogen \\
\hline DH5a $\lambda$ pir & supE44 $\triangle l a c U 169$ ( $(80$ lacZUM15) hsdR17 LAMpir U169 recA1 endA1 gyrA96 thi-1 relA1 & Lab stock \\
\hline W3110 & Wide type, $\lambda$-F-mcrA mcrB IN (rrnD-rrnE)1 & DSM5911 \\
\hline EC101 & W3110 with plasmid pET-trc-IEB & This study \\
\hline EC102 & W3110 with plasmid pCDF-yciG-IEB & This study \\
\hline EC103 & W3110 with plasmid pCDF-pstA-IEB & This study \\
\hline EC104 & W3110 with plasmid pCDF-yodA-IEB & This study \\
\hline EC105 & W3110 with plasmid pCDF-astC-IEB & This study \\
\hline EC106 & W3110 with plasmid pCDF-ybiM-IEB & This study \\
\hline EC201 & W3110 with plasmid pACYC-phnl & This study \\
\hline EC202 & W3110 with plasmid pACYC-phoR & This study \\
\hline EC203 & W3110 with plasmid pACYC-phnF & This study \\
\hline EC204 & W3110 with plasmid pACYC-phnC & This study \\
\hline EC205 & W3110 with plasmid pACYC-phnD & This study \\
\hline EC301 & W3110 with plasmid pACYC-yfiL & This study \\
\hline EC302 & W3110 with plasmid pACYC-yijF & This study \\
\hline EC303 & W3110 with plasmid pACYC-cysP & This study \\
\hline EC304 & W3110 with plasmid pACYC-yejG & This study \\
\hline EC305 & W3110 with plasmid pACYC-yhcN & This study \\
\hline EC401 & DH5a $\lambda$ pir with plasmid pRC-IS5 & This study \\
\hline EC-IS5 & W3110 with the integration of plasmid pRC-IS5 & This study \\
\hline $\mathrm{EC}-\mathrm{IS} 5(\Delta \mathrm{Cm})$ & EC-IS5 with the deletion of $\mathrm{Cm}$ & This study \\
\hline \multicolumn{3}{|l|}{ Plasmids $^{\mathrm{a}}$} \\
\hline pCDFDuet-1 & pCloDF13-derived vector; T7 promoter, $\operatorname{Str}^{R}$ & Lab stock \\
\hline pET-30a-trc & pBR322-derived vector; trc promoter, $\mathrm{Kmr}^{\mathrm{R}}$ & Lab stock \\
\hline pACYCDuet-1 & p15A-derived vector; $\mathrm{T7}$ promoter, $\mathrm{Cmr}^{\mathrm{R}}$ & Lab stock \\
\hline pTrc99a & pBR322-derived vector; trc promoter, Amp ${ }^{R}$ & [26] \\
\hline pRC43 & Including R6K ori, Cm, IS5 sequence & [29] \\
\hline pCas & repA101(Ts) kan Pcas-cas9 ParaB-Red lacla Ptrc-sgRNA-pMB1 & [14] \\
\hline pTargetF-cadA & pMB1 aadA sgRNA-cadA & [14] \\
\hline pET-trc-IEB & Lycopene synthetic module under the trc promoter & This study \\
\hline pCDF-yciG-IEB & Lycopene synthetic module under the yciG promoter & This study \\
\hline pCDF-pstA-IEB & Lycopene synthetic module under the pstA promoter & This study \\
\hline pCDF-yodA-IEB & Lycopene synthetic module under the yodA promoter & This study \\
\hline pCDF-astC-IEB & Lycopene synthetic module under the astC promoter & This study \\
\hline pCDF-ybiM-IEB & Lycopene synthetic module under the ybiM promoter & This study \\
\hline pACYC-phnl & Lycopene synthetic module under the yciG promoter, feedforward module under the phnl promoter & This study \\
\hline pACYC-phoR & Lycopene synthetic module under the yciG promoter, feedforward module under the phoR promoter & This study \\
\hline pACYC-phnF & Lycopene synthetic module under the yciG promoter, feedforward module under the phnF promoter & This study \\
\hline pACYC-phnC & Lycopene synthetic module under the yciG promoter, feedforward module under the phnC promoter & This study \\
\hline pACYC-phnD & Lycopene synthetic module under the yciG promoter, feedforward module under the phnD promoter & This study \\
\hline pACYC-yfiL & Lycopene synthetic module under the yciG promoter, feedback module under the yfiL promoter & This study \\
\hline pACYC-yijF & Lycopene synthetic module under the yciG promoter, feedback module under the yijF promoter & This study \\
\hline pACYC-cysP & Lycopene synthetic module under the yciG promoter, feedback module under the cysP promoter & This study \\
\hline pACYC-yejG & Lycopene synthetic module under the yciG promoter, feedback module under the yejG promoter & This study \\
\hline pACYC-yhcN & Lycopene synthetic module under the yciG promoter, feedback module under the yhcN promoter & This study \\
\hline pTargetF-delete & gRNA for N20PAM & This study \\
\hline pRC-IS5 & $\begin{array}{l}\text { Including R6K ori, } \mathrm{Cm} \text {, fragmentary IS5 sequence, Lycopene synthetic module under the trc promoter, feedforward } \\
\text { module under the phoR promoter and feedback module under the yejG promoter }\end{array}$ & This study \\
\hline
\end{tabular}

a $A m p^{R}$ : ampicillin; $\operatorname{Kan}^{\mathrm{R}}$ : kanamycin; $\operatorname{Str}^{\mathrm{R}}$ : Streptomycin; $\mathrm{Cm}$ : chloramphenicol; R:resistance 


\section{Supplementary information}

Supplementary information accompanies this paper at https://doi. org/10.1186/s12934-020-01360-x.

Additional file 1. Additional tables and figures.

\section{Acknowledgements}

Not applicable.

\section{Authors' contributions}

$\mathrm{HZ}$ conceived the project; BS designed the experiments; BS and DS performed the experiments; BS wrote and revised the manuscript. All authors read and approved the final manuscript.

\section{Funding}

Guangdong Province Science and Technology Innovation Strategy Special Fund (Grant No. 2018B020206001), GDAS'Special Project of Science and Technology Development (Grant No. 2020GDASYL-20200302002) and the Science and Technology Plan Project of Guangdong Province (2016A010105013, 2019B030316017).

\section{Availability of data and materials}

The datasets and materials used during the current study are available from the corresponding author on reasonable request.

\section{Ethics approval and consent to participate}

Not applicable.

\section{Consent for publication}

Not applicable.

\section{Competing interests}

The authors declare that they have no competing interests.

Received: 24 December 2019 Accepted: 30 April 2020

Published online: 24 May 2020

\section{References}

1. Yu T, Dabirian Y, Liu Q, Siewers V, Nielsen J. Strategies and challenges for metabolic rewiring. Curr Opin Syst Biol. 2019;15:30-8.

2. Pontrelli S, Chiu TY, Lan El, Chen FY, Chang P, Liao JC. Escherichia coli as a host for metabolic engineering. Metab Eng. 2018;50:16-46.

3. Ou B, Garcia C, Wang Y, Zhang W, Zhu G. Techniques for chromosomal integration and expression optimization in Escherichia coli. Biotechnol Bioeng. 2018;115:2467-78.

4. Pines G, Freed EF, Winkler JD, Gill RT. Bacterial recombineering: genome engineering via phage-based homologous recombination. ACS Synth Biol. 2015;4:1176-85.

5. Kuhlman TE, Cox EC. Site-specific chromosomal integration of large synthetic constructs. Nucleic Acids Res. 2010;38:e92.

6. Yang J, Sun B, Huang H, Jiang Y, Diao L, Chen B, Xu C, Wang X, Liu $J$, Jiang W, Yang S. High-efficiency scarless genetic modification in Escherichia coli by using lambda red recombination and I-Scel cleavage. Appl Environ Microbiol. 2014;80:3826-34.

7. Sabri S, Steen JA, Bongers M, Nielsen LK, Vickers CE. Knock-in/Knockout (KIKO) vectors for rapid integration of large DNA sequences, including whole metabolic pathways, onto the Escherichia coli chromosome at well-characterised loci. Microb Cell Fact. 2013;12:60.

8. Juhas M, Evans LD, Frost J, Davenport PW, Yarkoni O, Fraser GM, Ajioka JW. Escherichia coli flagellar genes as target sites for integration and expression of genetic circuits. PLoS ONE. 2014:9:e111451.

9. Juhas M, Ajioka JW. Lambda Red recombinase-mediated integration of the high molecular weight DNA into the Escherichia coli chromosome. Microb Cell Fact. 2016;15:172.
10. Jakociunas T, Jensen MK, Keasling JD. CRISPR/Cas9 advances engineering of microbial cell factories. Metab Eng. 2016;34:44-59.

11. Choi KR, Lee SY. CRISPR technologies for bacterial systems: current achievements and future directions. Biotechnol Adv. 2016:34:1180-209.

12. Chung ME, Yeh $I H$, Sung LY, Wu MY, Chao YP, Ng IS, Hu YC. Enhanced integration of large DNA into E. coli chromosome by CRISPR/Cas9. Biotechnol Bioeng. 2017;114:172-83.

13. Bassalo MC, Garst AD, Halweg-Edwards AL, Grau WC, Domaille DW, Mutalik VK, Arkin AP, Gill RT. Rapid and efficient one-step metabolic pathway integration in E. coli. ACS Synth Biol. 2016;5:561-8.

14. Jiang Y, Chen B, Duan C, Sun B, Yang J, Yang S. Multigene editing in the Escherichia coli genome via the CRISPR-Cas9 system. Appl Environ Microbiol. 2015;81:2506-14.

15. HamediRad M, Chao R, Weisberg S, Lian J, Sinha S, Zhao H. Towards a fully automated algorithm driven platform for biosystems design. Nat Commun. 2019;10:5150.

16. Chatzivasileiou AO, Ward V, Edgar SM, Stephanopoulos G. Twostep pathway for isoprenoid synthesis. Proc Natl Acad Sci USA. 2019:116:506-11.

17. Wang C, Zhao S, Shao X, Park JB, Jeong SH, Park HJ, Kwak WJ, Wei G, Kim SW. Challenges and tackles in metabolic engineering for microbial production of carotenoids. Microb Cell Fact. 2019;18:55

18. Saini RK, Keum YS. Microbial platforms to produce commercially vital carotenoids at industrial scale: an updated review of critical issues. J Ind Microbiol Biotechnol. 2019;46:657-74.

19. Mussagy CU, Winterburn J, Santos-Ebinuma VC, Pereira JFB. Production and extraction of carotenoids produced by microorganisms. Appl Microbiol Biotechnol. 2019;103:1095-114.

20. Niu FX, Lu Q, Bu YF, Liu JZ. Metabolic engineering for the microbial production of isoprenoids: carotenoids and isoprenoid-based biofuels. Synth Syst Biotechnol. 2017;2:167-75

21. Banerjee A, Sharkey TD. Methylerythritol 4-phosphate (MEP) pathway metabolic regulation. Nat Prod Rep. 2014;31:1043-55.

22. Dinh CV, Prather KLJ. Development of an autonomous and bifunctional quorum-sensing circuit for metabolic flux control in engineered Escherichia coli. Proc Natl Acad Sci USA. 2019;116:25562-8.

23. Gupta A, Reizman IM, Reisch CR, Prather KL. Dynamic regulation of metabolic flux in engineered bacteria using a pathway-independent quorum-sensing circuit. Nat Biotechnol. 2017;35:273-9.

24. George KW, Thompson MG, Kim J, Baidoo EEK, Wang G, Benites VT, Petzold CJ, Chan LJG, Yilmaz S, Turhanen P, et al. Integrated analysis of isopentenyl pyrophosphate (IPP) toxicity in isoprenoid-producing Escherichia coli. Metab Eng. 2018;47:60-72.

25. Sharon E, Kalma Y, Sharp A, Raveh-Sadka T, Levo M, Zeevi D, Keren $L$, Yakhini Z, Weinberger A, Segal E. Inferring gene regulatory logic from high-throughput measurements of thousands of systematically designed promoters. Nat Biotechnol. 2012;30:521-30.

26. Su B, Wu M, Zhang Z, Lin J, Yang L. Efficient production of xylitol from hemicellulosic hydrolysate using engineered Escherichia coli. Metab Eng. 2015:31:112-22

27. Wei Y, Mohsin A, Hong Q, Guo M, Fang H. Enhanced production of biosynthesized lycopene via heterogenous MVA pathway based on chromosomal multiple position integration strategy plus plasmid systems in Escherichia coli. Bioresour Technol. 2018:250:382-9.

28. Gibson DG, Young L, Chuang RY, Venter JC, Hutchison CA 3rd, Smith HO. Enzymatic assembly of DNA molecules up to several hundred kilobases. Nat Methods. 2009;6:343-5.

29. Su B, Zhang Z, Wu M, Lin J, Yang L. Construction of plasmid-free Escherichia coli for the production of arabitol-free xylitol from corncob hemicellulosic hydrolysate. Sci Rep. 2016;6:26567

30. Alonso-Gutierrez J, Koma D, Hu Q, Yang Y, Chan LJG, Petzold CJ, Adams PD, Vickers CE, Nielsen LK, Keasling JD, Lee TS. Toward industrial production of isoprenoids in Escherichia coli: lessons learned from CRISPRCas9 based optimization of a chromosomally integrated mevalonate pathway. Biotechnol Bioeng. 2018;115:1000-13.

31. Wang G, Shi T, Chen T, Wang X, Wang Y, Liu D, Guo J, Fu J, Feng L, Wang Z, Zhao X. Integrated whole-genome and transcriptome sequence analysis reveals the genetic characteristics of a riboflavin-overproducing Bacillus subtilis. Metab Eng. 2018;48:138-49. 
32. Bell JC, Kowalczykowski SC. RecA: regulation and mechanism of a molecular search engine. Trends Biochem Sci. 2016;41:491-507.

33. Li Y, Yan F, Wu H, Li G, Han Y, Ma Q, Fan X, Zhang C, Xu Q, Xie X, Chen N. Multiple-step chromosomal integration of divided segments from a large DNA fragment via CRISPR/Cas9 in Escherichia coli. J Ind Microbiol Biotechnol. 2019;46:81-90.

34. Li Y, Lin Z, Huang C, Zhang Y, Wang Z, Tang YJ, Chen T, Zhao X. Metabolic engineering of Escherichia coli using CRISPR-Cas9 meditated genome editing. Metab Eng. 2015:31:13-21.

35. Mahillon J, Chandler M. Insertion sequences. Microbiol Mol Biol Rev. 1998:62:725-74.

36. Sharan SK, Thomason LC, Kuznetsov SG, Court DL. Recombineering: a homologous recombination-based method of genetic engineering. Nat Protoc. 2009;4:206-23.
37. Zhu F, Lu L, Fu S, Zhong X, Hu M, Deng Z, Liu T. Targeted engineering and scale up of lycopene overproduction in Escherichia coli. Process Biochem. 2015;50:341-6.

38. Scott KJ. Detection and measurement of carotenoids by UVNIS spectrophotometry. Curr Protoc Food Anal Chem. 2001. https://doi. org/10.1002/0471142913.faf0202s00.

\section{Publisher's Note}

Springer Nature remains neutral with regard to jurisdictional claims in published maps and institutional affiliations.
Ready to submit your research? Choose BMC and benefit from:

- fast, convenient online submission

- thorough peer review by experienced researchers in your field

- rapid publication on acceptance

- support for research data, including large and complex data types

- gold Open Access which fosters wider collaboration and increased citations

- maximum visibility for your research: over $100 \mathrm{M}$ website views per year

At BMC, research is always in progress.

Learn more biomedcentral.com/submissions 